\title{
Rapid organism identification from Bactec NR blood culture media in a diagnostic microbiology laboratory
}

\author{
P M Claxton, R G Masterton
}

\begin{abstract}
Aims-To evaluate rapid organism identification on positive blood culture Bactec NR media (phial types 26, 27, 42 and 17), and to assess the usefulness of these procedures in a diagnostic microbiology laboratory.

Methods-Two hundred and sixty, first positive, blood culture bottles from individual patients were tested by rapid identification methods selected on the basis of Gram film organism morphology. Tube coagulase and latex agglutination were applied to presumptive staphylococci; latex agglutination antigen detection methods to suspected pneumococci, Neisseria and Haemophilus sp; and latex agglutination grouping tests for cultures thought to be non-pneumococcal streptococci.
\end{abstract}

Results-Media type did not influence test performance ( $p>0.05$ for all comparisons). Misapplication of methods occurred on eight occasions and there were 14 false positive results, nine involving the latex reagents for group $C$ streptococci and pneumococci. The positive predictive values for tube coagulase tests and latex reactions for $H$ influenzae type $b$, and $\boldsymbol{N}$ meningitidis groups $B$ and $C$ were $100 \%$. The pneumococcal and staphylococcal latex tests gave positive predictive values of $94 \cdot 1 \%$ and $62 \cdot 5 \%$, respectively, and the corresponding figure for streptococcal grouping reactions was $\mathbf{7 5 \cdot 9 \%}$. With the exception of staphylococcal latex testing (80\%) all investigation negative predictive values were $>90 \%$.

Conclusions-The performance of the staphylococcal latex agglutination method was unsatisfactory and it is not appropriate for use with the media studied. In view of the cross-reactions observed with the tests used to identify group C streptococci and pneumococci, positive findings must be interpreted with caution. In all other regards the protocol evaluated produced rapid, reliable, clinically useful information and, subject to local experience, is recommended to users of Bactec NR media.

(F Clin Pathol 1994;47:796-798)

Automated blood culture systems and improved blood culture media permit early detection of bacterial growth. However, the potential clinical benefits of this may be limited in practice as conventionally presumptive organism identification relies on Gram film morphology until culture becomes available, often 18-24 hours later.

Many workers have therefore used a variety of methods ${ }^{1-4}$ to explore rapid organism identification from positive blood culture bottles. These studies have evaluated the performances of specific tests rather than the utility of the approach in general. Only small numbers of blood cultures were included and results have been shown to be influenced by the type of media used. ${ }^{1}$

The published work has not included assessment of rapid identification methods in the recently available Bactec NR Plus media. Therefore, this study was designed to evaluate rapid organism identification using the new media, and to assess the usefulness of this approach in a diagnostic microbiology laboratory.

\section{Methods}

Adult patient blood culture sets comprised a pair of aerobic and anaerobic Bactec NR Plus media with paediatric samples made up of a Bactec NR Peds Plus bottle and an NR anaerobic bottle (phial types 26, 27, 42 and 17, respectively). The first positive blood culture bottle from any patient was included in the study.

The procedure for carrying out direct tube coagulase tests and staphylococcal and streptococcal latex agglutination reactions involved preparing an organism suspension by double centrifugation. ${ }^{12}$ Between $8-10 \mathrm{ml}$ of the positive blood culture was centrifuged at $700 \mathrm{rpm}$ for 10 minutes to remove the red blood cells. The supernatant fluid was recovered and centrifuged at $3000 \mathrm{rpm}$ for a further 10 minutes. The resultant deposit was re suspended in 0.5 $\mathrm{ml}$ sterile saline to produce the in-use suspension. ${ }^{12}$

For latex agglutination antigen detection of Streptococcus pneumoniae, Neisseria meningitidis, and Haemophilus influenzae, $2 \mathrm{ml}$ of the positive blood culture was centrifuged at 3000 rpm for 10 minutes and the supernatant fluid used as the test material. ${ }^{3}$

The rapid organism identification methods used on each positive blood culture bottle were determined by the organism morphology on the Gram film. Where relevant all methods thought to be appropriate were applied. Cultures thought to be mixed were excluded from the study.

Correspondence to: 
Table 1 Rapid organism identification by culture medium type

\begin{tabular}{|c|c|c|c|c|c|c|}
\hline \multirow[b]{2}{*}{ Culture identification } & \multirow[b]{2}{*}{$n=$} & \multicolumn{4}{|c|}{ Phial types } & \multirow{2}{*}{$\begin{array}{l}\text { No correctly identified } \\
\text { by rapid methods }\end{array}$} \\
\hline & & 26 & 27 & 42 & 17 & \\
\hline Haemophilus influenzae type $b$ & 19 & 2 & 0 & 17 & 0 & 16 \\
\hline Neisseria mengitidis group B & 7 & 0 & 0 & 7 & 0 & 7 \\
\hline Neisseria mengitidis group C & 2 & 0 & 0 & 2 & 0 & 2 \\
\hline Streptococcus pneumoniae & 66 & 41 & 9 & 8 & 8 & $64^{\star}$ \\
\hline \multicolumn{7}{|l|}{ Coagulase negative staphylococci } \\
\hline tube coagulase & 96 & 55 & 7 & 29 & 5 & 96 \\
\hline latex agglutination & 67 & 45 & 4 & 15 & 3 & 64 \\
\hline \multicolumn{7}{|l|}{ Staphylococcus aureus } \\
\hline tube coagulase & 29 & 24 & 2 & 2 & 1 & 19 \\
\hline latex agglutination & 21 & 17 & 1 & 3 & 0 & 5 \\
\hline Enterococcus sp & 15 & 7 & $i$ & 5 & 2 & $15 \#$ \\
\hline Group A streptococcus & 5 & 2 & 0 & 2 & 1 & 25 \\
\hline Group B streptococcus & 2 & 0 & 0 & 2 & 0 & 2 \\
\hline Group C streptococcus & 1 & 0 & 1 & 0 & 0 & $1+$ \\
\hline Group G streptococcus & 2 & 1 & 1 & 0 & 0 & 2 \\
\hline Non-groupable streptococci & 8 & 2 & 0 & 5 & 1 & $7 \ddagger$ \\
\hline
\end{tabular}

$\mathrm{n}=$ total number of isolates cultures

^Two not tested with pneumococcal latex reagent but identified as group C streptococci. Additionally, three other pneumococcal latex positive isolates cross-reacted with group $C$ streptococcal latex suspension.

\# One isolate cross-reacted with pneumococcal latex reagent

$\$$ Three not tested directly with group A streptococcal reagent

This isolated cross-reacted in the pneumococcal latex reagent

fOne isolate was not tested directly with streptococcal grouping suspensions, but gave a false positive pneumococcal test. isolates of $S$ pneumoniae, $N$ meningitidis, and $H$ influenzae were confirmed by reference laboratories.

Statistical analyses were performed using Fisher's exact probability test. The sensitivities, specificities, and predictive values of the different methods were calculated using standard formulae. ${ }^{6}$

\section{Results}

A total of 260 positive blood cultures were evaluated. One culture yielded mixed growth. The results of rapid organism identification by culture medium type are shown in table 1 . A statistical analysis of the true and false reactions by phial type was performed for staphylococcal coagulase, pneumococcal, and staphylococcal latex tests as there were insufficient numbers of the other organisms for valid comparisons (two tailed Fisher's exact test; $\mathrm{p}>0.05$ for all evaluations).

Table 2 shows the results for the individual investigations. The combined findings of the streptococcal grouping reactions produced a sensitivity of $100 \%$, a specificity of $50 \%$, and positive and negative predictive values of $75.9 \%$ and $100 \%$, respectively. There were eight misapplications of testing methods involving three staphylococcal and five streptococcal isolates. Of these, two staphylococci produced false positive group D streptococcal reactions, and two pneumococci were positive with group $\mathrm{C}$ streptococcus reagent but were not tested against pneumococcal latex suspension. Three group A streptococci were negative to pneumococcal agglutination but were not investigated with streptococcal grouping agent. The final misapplication involved a coagulase negative Staphylococcus which was thought morphologically to be a Streptococcus and was investigated as such, being negative to pneumococcal and streptococcal grouping reagents.

There were 14 false positive results. A mixed culture, an Enterococcus $s p$, a group C Streptococcus, and a non-groupable Streptococcus cross-reacted with pneumococcal latex reagent. Five pneumococci gave false positive group C streptococcal reactions. Three of these strains agglutinated pneumococcal latex suspension, the other was tested only with streptococcal grouping reagents. Two staphylococci gave false positive group D streptococ-

Table 2 Individual test performances

\begin{tabular}{|c|c|c|c|c|c|c|c|c|c|}
\hline Test & $n=$ & $T P$ & $T N$ & $F P$ & $F N$ & \% Sensitivity & $\%$ Specificity & $P P V$ & $N P V$ \\
\hline Tube coagulase & 125 & 19 & 96 & 0 & 10 & $65 \cdot 5$ & 100 & 100 & $90 \cdot 6$ \\
\hline Staphylococcus latex & 88 & 5 & 64 & 3 & 16 & $23 \cdot 8$ & $95 \cdot 5$ & $62 \cdot 5$ & $80 \cdot 0$ \\
\hline$H$ influenzae type b latex & 19 & 16 & N/A & 0 & 3 & $84 \cdot 2$ & N/A & 100 & N/A \\
\hline$N$ meningitidis group B latex & 9 & 7 & 2 & 0 & 0 & 100 & 100 & 100 & 100 \\
\hline$N$ meningitidis group $\mathrm{C}$ latex & 9 & 2 & 7 & 0 & 0 & 100 & 100 & 100 & 100 \\
\hline$S$ pneumoniae latex & 88 & 64 & 20 & 4 & 0 & 100 & $83 \cdot 3$ & $94 \cdot 1$ & 100 \\
\hline Group A streptococcus & 36 & 2 & 34 & 0 & 0 & 100 & 100 & 100 & 100 \\
\hline Group B streptococcus & 36 & 2 & 34 & 0 & 0 & 100 & 100 & 100 & 100 \\
\hline Group C streptococcus & 36 & 1 & 30 & 5 & 0 & 100 & $85 \cdot 7$ & $16 \cdot 7$ & 100 \\
\hline Group G streptococcus & 36 & 2 & 34 & 0 & 0 & 100 & 100 & 100 & 100 \\
\hline Enterococcus sp & 36 & 15 & 19 & 2 & 0 & 100 & 90.5 & $88 \cdot 2$ & 100 \\
\hline
\end{tabular}

$\mathrm{n}=$ number of test performances; TP = number of true positives; $\mathrm{TN}=$ number of true negatives; $\mathrm{FP}=$ number of false positives; FN = number of false negatives; N/A = not applicable as no true negatives tested; $P P V=\%$ positive predictive value; NPV $=\%$ negative predictive value. 
cal reactions, and there were three false positive staphylococcal latex agglutinations.

\section{Discussion}

The purpose of rapid blood culture isolate identification is to produce the earliest possible clinically meaningful information and it is particularly relevant where the data can be used to guide management. To this end most of the published work has investigated staphylococci and streptococci, especially pneumococci, with some attention also to Neisseria meningitidis and Haemophilus influenzae. With the exception of one small study, Gram negative bacilli in general have not been explored. ${ }^{2}$ This probably reflects less pressing diagnostic needs and the lack of influence such results are likely to have on treatment where antibiotic modification usually awaits sensitivity testing.

The performance of rapid blood culture isolate testing has limitations. The manufacturers of streptococcal latex agglutination products all recommend that to minimise false reactions the method should only be applied to $\beta$ haemolytic cultures. This is impossible when using the tests for direct investigations. Furthermore, organism identification is normally performed only on pure cultures and where initial investigations have indicated the genus. In rapid blood culture identifications the former cannot be guaranteed and the latter is based on Gram film morphology alone. Additionally, the ability to produce rapid results would be undermined if all possible tests were applied to each isolate, a protocol which in any event would be wasteful of human and consumable resources.

Our findings reinforce previous work on $N$ meningitidis and $H$ influenzae, ${ }^{23}$ showing rapid testing to be highly reliable with all positive results confirmed and only three false negative reactions with strains of $H$ influenzae type b. The situation is less satisfactory with $S$ pneumoniae where the problem of cross-reaction with group C streptococcal reagent is well recognised. In one study this reached $92 \%$ $(11 / 12)$ of clinical blood cultures tested. ${ }^{7}$ Using our selective protocol where not all pneumococci were examined with streptococcal grouping reagent, at $7 \cdot 6 \%(5 / 66)$ of confirmed identifications, our cross-reaction rate was much lower but still troublesome. Gram film morphology and blood lysis in bottles provided useful supporting identification information. Conversely, when applied to the non-pneumococcal streptococci, there was a $18 \cdot 2 \%(4 / 22)$ false positive reaction rate for pneumococcal antigen detection. Inevitably, given the comparatively low incidence of group C streptococcal bacteraemia, this performance makes the test positive predictive value for that organism very poor. However, in the main the direct application of latex grouping methods proved satisfactory in terms of identifying $\beta$ haemolytic streptococci and enterococci as well as in excluding nongroupable strains.

Rink ${ }^{2}$ commented on the poor performance of the staphylococcal latex identification method applied directly to blood cultures and Rappaport et $a l^{1}$ reported $77 \cdot 3 \%$ sensitivity and $100 \%$ specificity for the same reagent used in this study. Our findings indicate it to be less reliable and we recommend that it should not be used as a rapid method with Bactec NR media. A positive tube coagulase is, however, highly predictive, though negative reactions still leave a $10 \%$ chance of the organism being $S$ aureus.

Despite the limitations inherent in rapid identification of isolates in positive blood cultures, we have shown that the selection of tests based on Gram film morphology and the application of such methods as primary investigations to be robust tools. Although small organism numbers prevent firm conclusions, no media effect was observed. Rapid clinically useful information is produced cost effectively and, while recognising local experience to be essential, we recommend these procedures to any diagnostic laboratory using Bactec NR media.

We are grateful to Murex Ltd for their donation of the Staphaurex used in the trial.

1 Rappaport T, Sawyer KP, Nachamkin I. Evaluation of several commercial biochemical and immunologic methods for rapid identification of Gram-positive cocci directly from blood cultures. 7 Clin Microbiol 1988;26:1335-8.

2 Rink MJ. Rapid identification of organisms from blood cultures. Med Lab Sci 1990;41:6-9.

3 Williams RG, Hart CA. Rapid identification of bacterial antigen in blood cultures and cerebrospinal fluid. $f$ Clin Pathol 1988;41:691-3.

4 Wellstood S. Evaluation of Phadebact and Streptex kits for rapid grouping of streptococci directly from blood cul rapid grouping of streptococci directly
tures. $\mathcal{F}$ Clin Microbiol 1982;15:226-30.

5 Rossney AS, English LF, Keane CT. Coagulase testing compared with commercial kits for routinely identifying Staphylococcus aureus. F Clin Pathol 1990;43:246-52.

6 Strike PW. Test interpretation. In: Statistical methods in laboratory medicine. Oxford: Butterworth-Heinemann Ltd, 1991:336-46.

7 Lee P, Wetherall BL. Cross-reaction between Streptococcus pneumoniae and Group C Streptococcal latex reagent. $\mathcal{f}$ Clin Microbiol 1987;25:152-3. 